\title{
Combined road prediction and target tracking in collision avoidance
}

\author{
Andreas Eidehall \\ Vehicle Dynamics and Active Safety \\ Volvo Car Corporation \\ SE-405 31 Göteborg, Sweden \\ aeidehal@volvocars.com
}

\author{
Fredrik Gustafsson \\ Department of Electrical Engineering \\ University of Linköping \\ SE-581 83 Linköping, Sweden \\ fredrik@isy.liu.se
}

\begin{abstract}
Detection and tracking of other vehicles and lane geometry will be required for many future intelligent driver assistance systems. By integrating the estimation of these two features into a single filter, a more optimal utilization of the available information can be achieved. For example, it is possible to improve the lane curvature estimate during bad visibility by studying the motion of other vehicles.
\end{abstract}

This paper derives and evaluates various approximations that are needed in order to deal with the non-linearities that are introduced by such an approach.

\section{Introduction}

Future intelligent automotive systems such as adaptive cruise control, collision avoidance or lane guidance will require detailed knowledge about the vehicle surroundings [6]. In this paper, vehicle surroundings will refer to lane geometry and other vehicles. Typically, lane information is obtained from a vision system and other vehicles are detected with a radar.

The importance of integrating data from object tracking and road geometry tracking has quite recently been recognized [3], [2], [7]. The main idea is to try to improve the road geometry estimate by studying the motion of other vehicles and vice versa. For example, if a couple of tracked vehicles suddenly all start moving right, one of two things can have happened. The first is that they all started a lane change manoeuvre and the road remains straight. The other is that we are entering a curve and the vehicles are still following the center of their lanes. These possibilities can be treated in a Bayesian framework, together with the information from the lane tracker, to build a new estimator. In order to do this we need to construct a new object measurement equation based on the road geometry.

\section{Overview}

The position on the road for each vehicle is denoted $(x, y)$. This means that $x$ is the driven distance along the road and $y$ the lateral position in the lane. We will need to relate this to absolute, or Cartesian, position denoted $(\tilde{x}, \tilde{y})$. The purpose of Section III-A is to find this transformation $(\tilde{x}, \tilde{y})=T(x, y)$. To derive this, we need a model of the road, and we start with a general model describing the road curvature as $c(x)=c_{0}+c_{1} x$. This describes a clothoid curve and is a commonly used parametrization in collision avoidance applications. The trigonometric formulas that arise do not give an explicit expression for $T(x, y)$. If such an expression is needed, either the trigonometric functions can be Taylor expanded, or a simpler model with $c_{1}=0$ can be used (constant curve radius). These approximations are treated in Section III-C.

To be able to apply a Kalman filter, we first define a state vector that contains road geometry, the host vehicle's and the tracked vehicles' positions. Section IV-A gives the measurement equations, where the host vehicle's sensors are expressed as functions of the state vector. A suitable motion model for the host vehicle and the tracked vehicles is suggested in Section IV-B.

Having said this, the results presented in Section V should be possible to appreciate, without understanding all of the detail in the model and filter described in Section III and IV.

\section{Measurement equation derivation}

\section{A. Coordinate system derivation}

We will start by deriving a two dimensional coordinate transformation which is a mapping $T$ from a curved coordinate system $(x, y)$ which follows the road to a cartesian coordinate system $(\tilde{x}, \tilde{y})$ which is attached to the host vehicle, see Figure 1. The first part of the derivation is similar to what was done in [4].

We start with a planar curve $\mathbf{r}(x)$, where $x$ is distance along a curve. Assume the curvature along the curve is given by $c=c(x)$. Now, if $\hat{\mathbf{t}}(x)$ is the tangent vector, where 


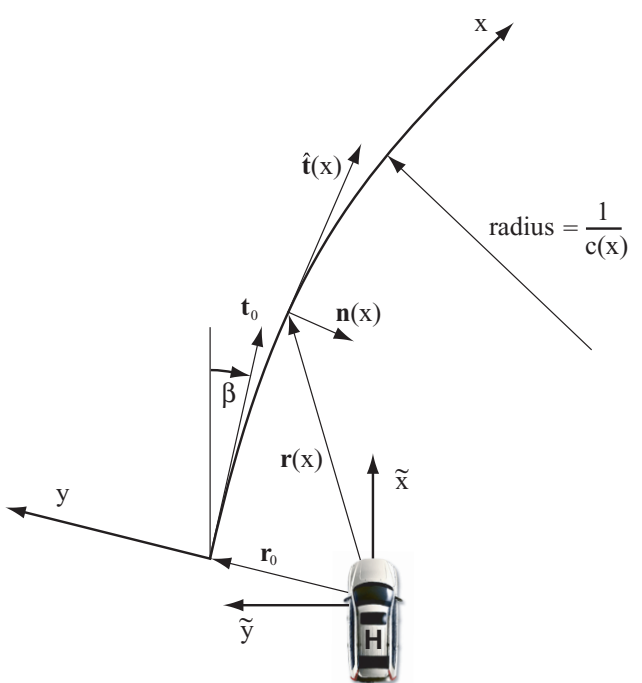

Fig. 1. The vectors used in the derivation of $T$.

the hat means $|\hat{\mathbf{t}}(x)|=1$, we define the normal vector $\mathbf{n}(x)$ as

$$
\mathbf{n}(x)=\frac{d \hat{\mathbf{t}}}{d x}(x)
$$

From vector analysis we get an alternative expression for $\mathbf{n}(x)$ where we use the fact that it is perpendicular to $\hat{\mathbf{t}}(x)$ and that its length is precisely $c(x)$. We first define

$$
R(\alpha) \triangleq\left(\begin{array}{cc}
\cos \alpha & -\sin \alpha \\
\sin \alpha & \cos \alpha
\end{array}\right)
$$

Then $\mathbf{n}(x)=c(x) R\left(-\frac{\pi}{2}\right) \hat{\mathbf{t}}(x)$. Thus, we end up with the differential equation

$$
\frac{d \hat{\mathbf{t}}}{d s}(x)=c(x) R\left(-\frac{\pi}{2}\right) \hat{\mathbf{t}}(x)
$$

which has the solution

$$
\hat{\mathbf{t}}(x)=\exp \left(R\left(-\frac{\pi}{2}\right) \int_{0}^{x} c(\tau) d \tau\right) \hat{\mathbf{t}}_{0}=R\left(-\int_{0}^{x} c(\tau) d \tau\right) \hat{\mathbf{t}}_{0}
$$

This can then be used to obtain an expression for the position:

$$
\begin{aligned}
\mathbf{r}(x) & =\int_{0}^{x} \hat{\mathbf{t}}(\tau) d \tau+\mathbf{r}_{0}= \\
& =\int_{0}^{x} R\left(-\int_{0}^{\tau_{1}} c\left(\tau_{2}\right) d \tau_{2}\right) d \tau_{1} \hat{\mathbf{t}}_{0}+\mathbf{r}_{0}
\end{aligned}
$$

The vectors $\mathbf{r}(x)$ and $\hat{\mathbf{t}}(x)$ are illustrated in Figure 1.

To construct the coordinate transformation $T$ we first define one of our coordinates to be the distance along the curve: $T(x, 0)=\mathbf{r}(x)$. To define the other coordinate we require $T$ to be orthogonal, i.e.

$$
\frac{d T(x, y)}{d y} \perp \hat{\mathbf{t}}(x) \text { for all } x
$$

A natural choice is to simply extend a straight line at $T(x, 0)$ along $-R\left(-\frac{\pi}{2}\right) \hat{\mathbf{t}}(x)$. This choice will also give us a positively oriented transformation. $T$ will look like

$$
\left(\begin{array}{c}
\tilde{x} \\
\tilde{y}
\end{array}\right)=T(x, y)=\mathbf{r}(x)-R\left(-\frac{\pi}{2}\right) \hat{\mathbf{t}}(x) y
$$

\section{B. Choosing curvature function}

The road is often modelled as segments of straight lines, arcs and clothoids, see for example [4]. Clothoids are segments were the curvature changes linearly with the distance along the curve. According to [1], this agrees well with how roads are constructed. The function

$$
c(x)=c_{0}+c_{1} x
$$

will suffice for all these cases. Of course, when approaching a curve we might, for example, have situations were the section 0 - 50 meters of our field of view is a straight line and the section 50 - 100 meters is a clothoid, a case which can not be modelled with a linear curvature law. Plugging (4) into (1) and using

$$
\hat{\mathbf{t}}_{0}=\left(\begin{array}{c}
\cos \beta \\
\sin \beta
\end{array}\right)
$$

we get

$$
\begin{aligned}
\hat{\mathbf{t}}(x) & =R\left(-c_{0} x-c_{1} x^{2} / 2\right)\left(\begin{array}{c}
\cos \beta \\
\sin \beta
\end{array}\right)= \\
& =\left(\begin{array}{cc}
\cos \left(c_{0} x+c_{1} x^{2} / 2\right) & \sin \left(c_{0} x+c_{1} x^{2} / 2\right) \\
-\sin \left(c_{0} x+c_{1} x^{2} / 2\right) & \cos \left(c_{0} x+c_{1} x^{2} / 2\right)
\end{array}\right)\left(\begin{array}{c}
\cos \beta \\
\sin \beta
\end{array}\right) \\
& =\left(\begin{array}{c}
\cos \beta \cos \left(c_{0} x+c_{1} x^{2} / 2\right)+\sin \beta \sin \left(c_{0} x+c_{1} x^{2} / 2\right) \\
-\cos \beta \sin \left(c_{0} x+c_{1} x^{2} / 2\right)+\sin \beta \cos \left(c_{0} x+c_{1} x^{2} / 2\right)
\end{array}\right) \\
& =\left(\begin{array}{cc}
\cos \beta & -\sin \beta \\
\sin \beta & \cos \beta
\end{array}\right)\left(\begin{array}{c}
\cos \left(c_{0} x+c_{1} x^{2}\right) \\
-\sin \left(c_{0} x+c_{1} x^{2}\right)
\end{array}\right) \\
& =R(\beta)\left(\begin{array}{c}
\cos \left(c_{0} x+c_{1} x^{2}\right) \\
-\sin \left(c_{0} x+c_{1} x^{2}\right)
\end{array}\right)
\end{aligned}
$$

(5) gives the second term of (3). In order to get an expression for the first term we need to integrate (5), which can not be done analytically. Instead we need to do some sort of approximation.

Before we continue we shall use the free parameter $\mathbf{r}_{0}$ to describe an offset perpendicular to $\hat{\mathbf{t}}_{0}$ by simply setting

$\mathbf{r}_{0}=y_{\text {off }} R\left(-\frac{\pi}{2}\right) \mathbf{t}_{0}=y_{\text {off }}\left(\begin{array}{cc}0 & 1 \\ -1 & 0\end{array}\right)\left(\begin{array}{c}\cos \beta \\ \sin \beta\end{array}\right)=R(\beta)\left(\begin{array}{c}0 \\ -y_{\text {off }}\end{array}\right)$

The vector $\mathbf{r}_{0}$ is shown in Figure 1. 


\section{Approximations}

\section{Approximation A: Omitting the clothoid parameter}

Using $c_{1}=0$ in (5) we get

$$
\hat{\mathbf{t}}(x)=R(\beta)\left(\begin{array}{c}
\cos \left(c_{0} x\right) \\
-\sin \left(c_{0} x\right)
\end{array}\right)
$$

which, used in (2) gives

$$
\mathbf{r}(x)=R(\beta)\left(\begin{array}{c}
\sin \left(c_{0} x\right) \\
\cos \left(c_{0} x\right)-1
\end{array}\right) \frac{1}{c_{0}}-R(\beta)\left(\begin{array}{c}
0 \\
y_{\text {off }}
\end{array}\right)
$$

and, since rotations commute, the coordinate transformation (3) becomes

$$
\begin{aligned}
T_{a}(x, y) & \triangleq \mathbf{r}(x)-R\left(\frac{\pi}{2}\right) \mathbf{t}(x) y= \\
& =R(\beta)\left(\begin{array}{c}
\left(1+c_{0} y\right) \sin \left(c_{0} x\right) \\
\left(1+c_{0} y\right) \cos \left(c_{0} x\right)-1-c_{0} y_{\text {off }}
\end{array}\right) \frac{1}{c_{0}}
\end{aligned}
$$

Approximation B: Linearizing the trigonometric functions

If we use $\sin \tau=\tau$ and $\cos \tau=1$ then (5) becomes

$$
\hat{\mathbf{t}}(x)=R(\beta)\left(\begin{array}{c}
1 \\
-c_{0} x-c_{1} x^{2} / 2
\end{array}\right)
$$

Plugging this into (2) we get

$$
\mathbf{r}(x)=R(\beta)\left(\begin{array}{c}
x \\
-c_{0} x^{2} / 2-c_{1} x^{3} / 6
\end{array}\right)-R(\beta)\left(\begin{array}{c}
0 \\
y_{\text {off }}
\end{array}\right)
$$

and from (3) we get the coordinate transformation

$$
T_{b}(x, y) \triangleq R(\beta)\left(\begin{array}{c}
x+y\left(c_{0} x+c_{1} x^{2} / 2\right) \\
y-y_{o f f}-c_{0} x^{2} / 2-c_{1} x^{3} / 6
\end{array}\right)
$$

Approximation C: As B, plus further approximations

We now do some further approximation steps

$$
\begin{aligned}
& T_{b}(x, y) \approx R(\beta)\left(\begin{array}{c}
x \\
y-y_{\text {off }}-c_{0} x^{2} / 2-c_{1} x^{3} / 6
\end{array}\right) \approx \\
& \approx\left(\begin{array}{cc}
1 & -\beta \\
\beta & 1
\end{array}\right)\left(\begin{array}{c}
x \\
y-y_{\text {off }}-c_{0} x^{2} / 2-c_{1} x^{3} / 6
\end{array}\right)= \\
& =\left(\begin{array}{c}
x-\beta\left(y-y_{\text {off }}-c_{0} x^{2} / 2-c_{1} x^{3} / 6\right) \\
\beta x+y-y_{\text {off }}-c_{0} x^{2} / 2-c_{1} x^{3} / 6
\end{array}\right) \approx \\
& \approx\left(\begin{array}{c}
x \\
y-y_{\text {off }}+\beta x-c_{0} x^{2} / 2-c_{1} x^{3} / 6
\end{array}\right) \triangleq T_{c}(x, y)
\end{aligned}
$$

This is a commonly used approximation.

\section{Filter construction}

To be able to use a Kalman filter, we will build three state space models, based on the approximations from the previous section. The states for the host vehicle are shown in Figure 2. Note that we have $\beta=-\Psi_{\text {rel }}$. We need $c_{1}$ as state in all three filters for dynamic reasons. Observed vehicles will have the states $x_{i}, \dot{x}_{i}$ and $y_{i}$, where $i$ runs through all detected objects.

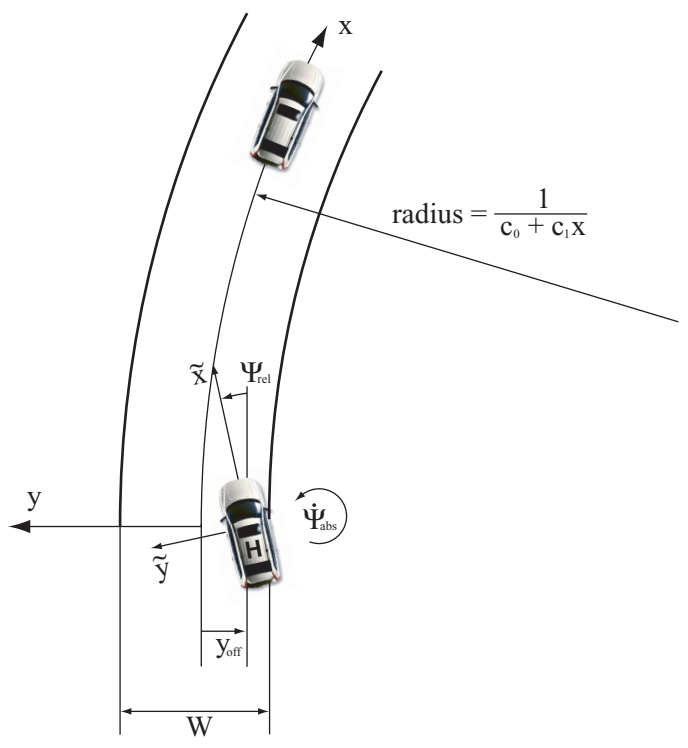

Fig. 2. $W, y_{\text {off }}, \Psi_{\text {rel }}, c_{0}$ and $c_{1}$ are the host vehicle states. The mapping $T$ transforms from the coordinate system $(x, y)$ to the coordinate system $(\tilde{x}, \tilde{y})$.

\section{A. Measurement equations}

The measurements for the host vehicle are $\Psi_{r e l}^{m}, c_{0}^{m}, L^{m}$ and $R^{m}$ where the last two are the distances to the left and right lane marking. Superscript $m$ denotes measured quantities. For other vehicles we measure the position, $\tilde{x}^{m}$ and $\tilde{y}^{m}$. These relate to the states as

$$
\begin{aligned}
L_{t}^{m} & =-W_{t} / 2-y_{o f f, t}+e_{1, t} \\
R_{t}^{m} & =W_{t} / 2-y_{o f f, t}+e_{2, t} \\
\Psi_{r e l, t}^{m} & =\Psi_{r e l, t}+e_{3, t} \\
c_{0, t}^{m} & =c_{0, t}+e_{4, t} \\
\left(\begin{array}{c}
\tilde{x}_{i, t}^{m} \\
\tilde{y}_{i, t}^{m}
\end{array}\right) & =T\left(x_{i, t}, y_{i, t}\right)+\left(\begin{array}{c}
e_{5, t} \\
e_{6, t}
\end{array}\right)_{i}
\end{aligned}
$$

where $T$ can be replaced with any of the approximations. Of course, $T$ depends on all the host vehicle states as well. The variables $\left(e_{1}, \ldots, e_{6}\right)$ are some stochastic measurement noise.

\section{B. Motion models}

Since $x$ and $y$ are the curved road coordinates, the motion model of other vehicles can be greatly simplified. For example, it allows us to use the equation $\dot{y}_{i}=0$ which simply means that we assume that other vehicles will follow their own lanes. In the longitudinal direction we will use the $\ddot{x}_{i}=0-a_{\text {host }} \cos \Psi_{\text {rel }}$, $a_{\text {host }}$ being the measured acceleration of the host vehicle so that with sample time 
$T_{S}$ we get the motion equations:

$$
\begin{aligned}
& x_{t+1}^{i}=x_{t}^{i}+T_{s} \dot{x}_{t}^{i}+a_{\text {host }, t} \cos \Psi_{r e l, t} T_{s}^{2} / 2+w_{1, t} \\
& \dot{x}_{t+1}^{i}=\dot{x}_{t}^{i}+a_{h o s t, t} \cos \Psi_{r e l, t} T_{s}+w_{2, t} \\
& y_{t+1}^{i}=y_{t}^{i}+w_{3, t}
\end{aligned}
$$

For the road geometry parameters we first clarify that $\Psi_{r e l}$ is the angle offset to the lane and $\Psi_{a b s}$ is the angle to some fix reference. We can obtain a relationship between the two by taking the time derivative of $\Psi_{\text {rel }}$

$$
\begin{aligned}
& \Psi_{r e l}=\Psi_{a b s}+\gamma \Rightarrow \\
& \dot{\Psi}_{r e l}=\dot{\Psi}_{a b s}+\dot{\gamma}=\dot{\Psi}_{a b s}+\frac{v}{r}=\dot{\Psi}_{a b s}+c_{0} v
\end{aligned}
$$

where $r$ is the current road radius, $v$ the velocity and $\gamma$ denotes the angle between the lane and some fix reference. $\dot{\Psi}_{a b s}$ can typically be measured with a yaw rate sensor. We also have

$$
\dot{y}_{\text {off }}=\sin \Psi_{r e l} v \approx \Psi_{r e l} v
$$

Using $\dot{W}=0$ and $\dot{c}_{1}=0$ we can write the motion equations for the host vehicle states:

$$
\begin{aligned}
W_{t+1} & =W_{t}+w_{4, t} \\
y_{o f f, t+1} & =y_{o f f, t}+v T_{s} \Psi_{r e l, t}+w_{5, t} \\
\Psi_{r e l, t+1} & =\Psi_{r e l, t}+v T_{s} c_{0, t}+T_{s} \dot{\Psi}_{a b s, t}+w_{6, t} \\
c_{0, t+1} & =c_{0, t}+v T_{s} c_{1, t}+w_{7, t} \\
c_{1, t+1} & =c_{1, t}+w_{8, t}
\end{aligned}
$$

The variables $\left(w_{1}, \ldots, w_{8}\right)$ are stochastic process noise.

\section{Evaluation}

\section{A. Geometric comparison}

From [1] we get the following guidelines for road construction. For a $50 \mathrm{~km} / \mathrm{h}$ road, the minimum radius is 140 meters and for a $90 \mathrm{~km} / \mathrm{h}$ road it is 550 meters. The recommended maximum clothoid parameters for these curves are given by the formula

$$
c_{1}=\frac{k}{v^{3}}
$$

where $k=0.45\left(\mathrm{~m} / \mathrm{s}^{3}\right)$ which is the maximum "jerk" and $v$ the velocity, giving the clothoid $1.7 \cdot 10^{-4}$ and $2.9 \cdot 10^{-5}\left(1 / \mathrm{m}^{2}\right)$ for the $50 \mathrm{~km} / \mathrm{h}$ and the $90 \mathrm{~km} / \mathrm{h}$ curve respectively. In Figure 3 we have compared the approximations with the exact transformation. Note that on straight lines and on circle segments, $T_{a}$ coincides with the exact transformation.

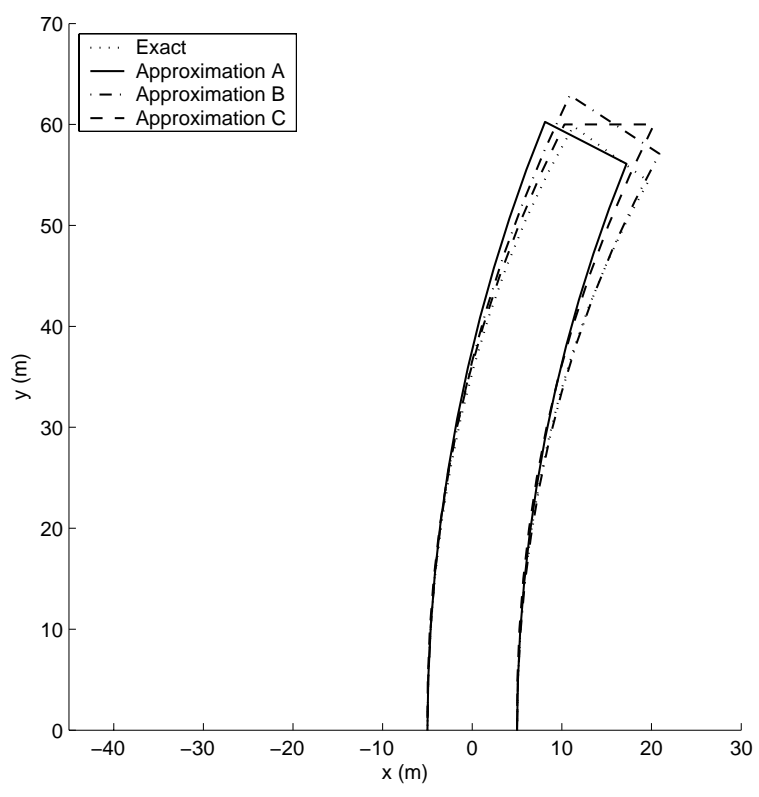

Fig. 3. Illustration of the different approximations. A road with edges at $y=-5$ and $y=5$ has been transformed with the three approximations and with the true transformation, based on typical a $50 \mathrm{~km} / \mathrm{h}$ curve.

\section{B. Curvature estimate accuracy}

To analyze the different models further, an extended Kalman filter was implemented, based on the different approximations. The accuracy of the lane geometry was then evaluated during a test drive. A vehicle equipped with a camera and a radar was used to record data. Lane geometry measurements were given by the camera and measurements of other vehicles were given both from the camera and the radar. Some additional data from the vehicle, such as velocity and yaw rate, were also collected. The true values of the lane geometry were obtained from a detailed map.

Four filters were then run on the same data, the three approximations that was derived in Section III-C, plus a completely decoupled model, i.e. tracking of obstacles and lane geometry done separately.

\section{Filter tuning}

Filter tuning is the process of adjusting the entries in the " $Q$ " and " $R$ " matrices, which are often interpreted as process and measurement noise covariance. If we constrain these to be diagonal, we have 14 parameters to tune, as shown in Table I.

The tuning was started by first using "physical" intuition trying to judge errors in measurements and changes in different states. This was then used as a starting point for hours of manual tuning in order to get acceptable performance from all three filters. 


\begin{tabular}{r|cc} 
& Host & Obstacles \\
\hline Process noise & 5 & 3 \\
Measurement noise & 4 & 2
\end{tabular}

TABLE I

NUMBER OF TUNING PARAMETERS

After that, a more systematic approach was used. A scaling parameter was applied to selected filter parameters. For example, if the host states process noise covariance matrix is called $Q_{\text {host }}$, the filters was run with this matrix replaced with $\lambda Q_{\text {host }}$, where $\lambda$ for example ranges from $10^{-2}$ to $10^{2}$. Then the mean error in some parameter, usually the curvature $c_{0}$, was computed. This procedure was then applied to different combinations of parameters or single parameters. This is of course only a suboptimization in the 14 dimensional parameter space.

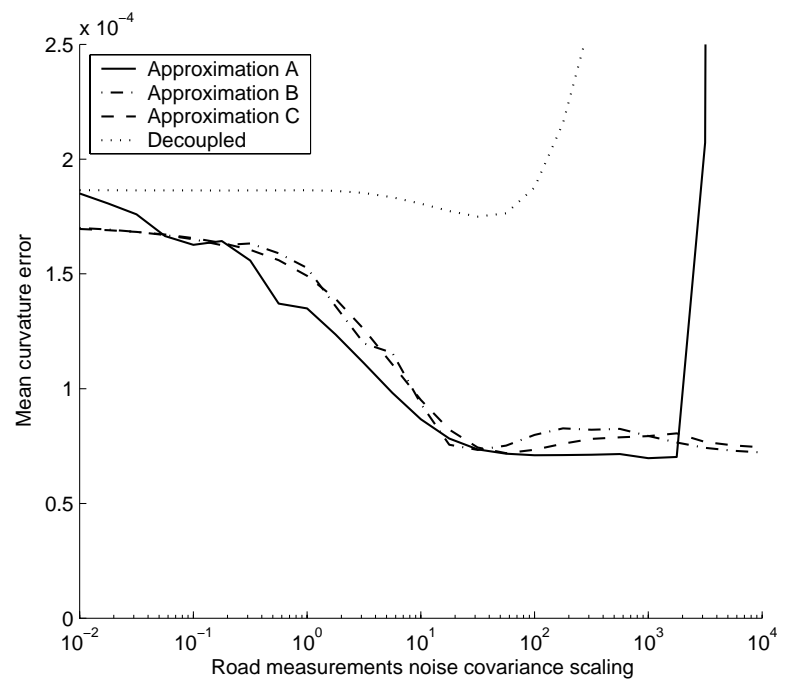

Fig. 4. Curvature error during bad visibility. The measurement noise of the road measurements has been scaled from $10^{-2}$ to $10^{4}$. The plot shows the error in the curvature estimate for the different filters. This also includes a decoupled filter where the road geometry and the obstacles are treated separately. Note that Approximation A becomes unstable for high measurement noise values.

\section{Results}

Figure 4 and Figure 5 shows some preliminary results of the performance of the different filters. The three approximations have been compared to a decoupled linear filter, where lane geometry and obstacles were tracked separately. Figure 4 shows a data sequence recorded during bad visibility. It shows that the performance can be improved by using a combined filter, which has also been demonstrated in for example [7] and [5]. Figure 5 shows a data set recorded during good visibility. In this case, there is only a small improvement by using integrated filtering.

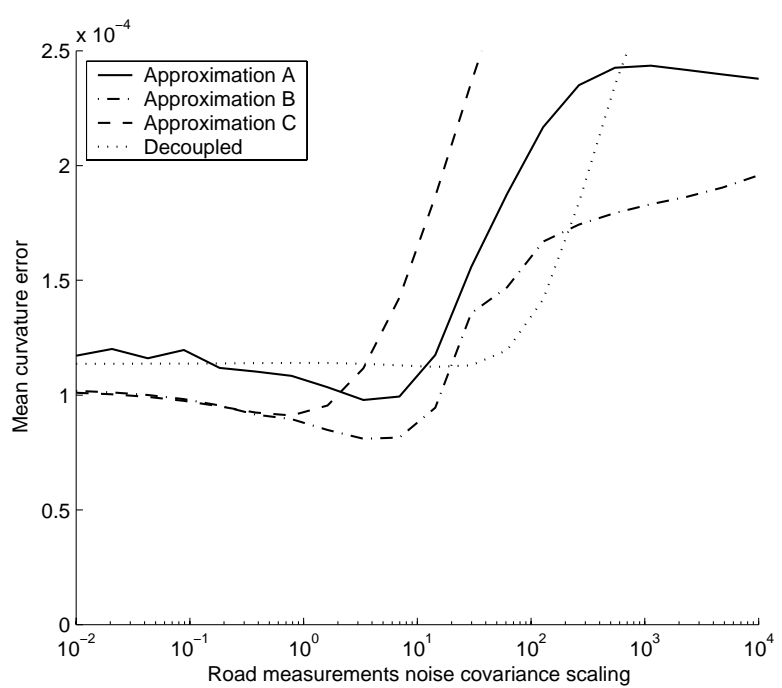

Fig. 5. Curvature error during good visibility. The measurement noise of the road measurements has been scaled from $10^{-2}$ to $10^{4}$. The plot shows the error in the curvature estimate for the different filters.

To make the experiment more interesting, we have only allowed the measurement noise of the road measurements to vary between the two cases, all other parameters are kept constant. It can be seen in the Figures 4 and 5 that the optimal performance is reached at higher measurement noise for the bad visibility case than the good visibility case. This is intuitive, if bad visibility was detected by for example the vision system, you would typically increase the process noise of road measurements in the Kalman filter in order to rely more on other measurements and on the motion model.

It should be noted that the data in Figure 4 and Figure 5 are from different roads and during different traffic conditions. Therefore, care should be taken before comparing the performance in the two experiments.

Also, even though the curvature is important in many applications, it is just one of many parameters. A more thorough evaluation of the performance could be done if the particular application of the filter was known.

In the next section we examine a property that is important in many collision avoidance applications.

\section{Lane assignment}

Lane assignment is the problem of deciding in which lanes the tracked vehicles are currently driving. This is where the quality of the lane geometry estimate becomes utterly important, even the slightest error in heading angle or curvature will result in a significant lateral error for vehicles at a long distance, say 70 - 100 meters.

All three model approximations from Section III-C were 


\begin{tabular}{r|c|c} 
Filter & Bad visibility & Good visibility \\
\hline Approximation A & 0.84 & 0.94 \\
Approximation B & 0.84 & 0.88 \\
Approximation C & 0.78 & 0.74 \\
Decoupled & 0.12 & 0.81 \\
\multicolumn{3}{c}{ TABLE II } \\
LANE ASSIGNMENT ACCURACY
\end{tabular}

run on the same data, again together with a decoupled filter. Doing lane assignment when using these filters becomes trivial due to the curved coordinate system. We only need to consider the lateral position of each estimate, i.e. the $y_{t}^{i}$ states, and the current lane width, $W_{t}$. We will refer to this quantity as the estimated lane index, $\hat{\chi}_{t}^{i}$. It is defined as

$$
\hat{\chi}_{t}^{i} \triangleq\left\{\begin{array}{rc}
-1 & y_{t}^{i}<-W_{t} / 2 \\
0 & -W_{t} / 2<y_{t}^{i}<W_{t} / 2 \\
+1 & W_{t} / 2<y_{t}^{i}
\end{array}\right.
$$

This value then needs to be compared to the true value, $\chi_{t}^{i}$. This was obtained by placing the measured position of other vehicles on the true map. This method is based on the assumption that the error in the position of the obstacles is small compared to the error in the lane geometry.

We then compute the accuracy of the lane assignment as the ratio of the correct number of assignments and the sum of the total number of obstacles summed over the entire data set, i.e. if $n_{\mathrm{tot}, t}$ is the total number of obstacles for time step $t$ and $n_{\text {correct. } t}$ is the number of correct assignments, we define

$$
\text { Lane assignment accuracy } \triangleq \frac{\sum_{t} n_{\text {correct }, t}}{\sum_{t} n_{\mathrm{tot}, t}}
$$

\section{Results}

The lane assignment accuracy of the different approximations from Section III-C are shown in Table II. It shows that the improvement achieved by integrated filtering during the bad visibility case is significant. In the case of good visibility, there is some improvement for Approximation $\mathrm{A}$ and $\mathrm{B}$.

\section{Summary and Conclusions}

It is clear that combined lane prediction and target tracking can give better estimates and improve the accuracy of state estimates and improve the performance of applications such as lane assignment. The integrated filter bring non-linearities which needs to be approximated, and it has been shown that there might be better alternatives than linearizing the trigonometric functions, which is often done.
Tuning the different filters is a very difficult task. The performance of all three filters can probably be improved if a more systematic tuning procedure is used. There are also stability issues, all three filters tend to diverge for certain, badly chosen, sets of tuning parameters. Stability of the extended Kalman filter can never be guaranteed.

Despite this, the improvement in performance shown in these experiments, cannot be overlooked, no matter which approximation is used.

\section{References}

[1] Vägutformning 94 version s-2, 2002:116. Technical report, Swedish National Road Administration, 1994.

[2] Automotive collision avoidance system field operational test. Technical report, National Highway Traffic Safety Administration, 2000.

[3] F. Dellaert and C. Thorpe. Robust car tracking using Kalman filtering and Bayesian templates. SPIE: Intelligent Transportation Systems, 1997.

[4] E. D. Dickmanns and A. Zapp. A curvature-based scheme for improving road vehicle guidance by computer vision. SPIE conference on mobile robots, 1986

[5] A. Gern, U. Franke, and P. Levi. Advanced lane recognition - fusing vision and radar. IEEE Intelligent Vehicles Symposium 2000, 2000.

[6] J. Jansson. Tracking and Decision Making for Automotive Collision Avoidance. Department of Electrical Engineering, Linköpings Universitet, 2002.

[7] Z. Zomotor and U. Franke. Sensor fusion for improved vision based lane recognition and object tracking with range-finders. IEEE Conference on intelligent transportation systems, 1997. 